\title{
Measurement: System, Uncertainty and Response
}

\author{
Md. Zahurul Haq* \\ Bangladesh University of Engineering \& Technology (BUET) \\ Bangladesh
}

\section{Introduction}

A measuring instrument transforms a measurand, i.e., a physical variable to be measured, to provide comprehensible output. An instrument can be aptly analysed and synthesized as a system which is a set of interconnected components functioning as a unit, while response is a measure of such unit's fidelity to its purpose. A real instrument's response is neither perfect nor identical even under static and replicate-conditions. So appropriate approach to quantify the deviation from the true value is required. It is now widely recognized that measurement results should be expressed in terms of estimated value and an associated uncertainty value obtained by proper analysis. System response analysis is further complicated in dynamic measurements as an instrument does not respond instantaneously to an input that varies in time. This obviously creates a measurement problem, and if these effects are not accounted for, dynamic errors are introduced. Therefore, the performance of a measuring instrument is to be specified in terms of both static and dynamic performance parameters.

\section{Measurement system}

Measurement is the act of assigning a specific value to a measurand. Mass, distance, time, temperature, force, and other physical quantities, as well as the properties of matters, materials, and devices, must be measured and described in common terminology. Measuring instruments are designed to generate a fixed and reproducible magnitude of the measurand which is expressed by a number (the magnitude ratio) followed by the matching unit, e.g., a length of $2.5 \mathrm{~m}$. So measurement provides quantitative information on the actual state of the measurand that otherwise could only be estimated. ISO/IEC Guide 98:2008 Uncertainty of Measurement - Guide to the Expression of Uncertainty in Measurement (GUM) (ISO, 2008) reports the following scope of applications of measurements:

- To maintain quality control and quality assurance in production;

- To comply with and enforcing laws and regulations;

- To conduct basic/applied research and development, in science and engineering;

- To develop, maintain and compare international and national physical reference standards, reference materials, and also to achieve traceability to national standards.

*http://teacher.buet.ac.bd/zahurul/ 
According to ISO/IEC Guide 99:2007 International Vocabulary of Metrology - Basic and General Concepts and Associated Terms (VIM) (ISO, 2007), measurement is defined as:

process of experimentally obtaining one or more quantity values that can reasonably be attributed to a quantity.

Thus measurement is an experimental science and most experiments are classified into following four categories (Dunn, 2010):

1. Variational experiments. These are carried out with an objective to establish the mathematical relations between the experiment's variables.

2. Validation experiments. These are carried out to validate a specific hypothesis.

3. Pedagogical experiments. These are aimed to demonstrate something that is already known.

4. Exploration experiments. These are conducted to explore an idea or possible theory.

\subsection{General measurement system}

It is sensible to have a generalized description of both the operation and performance of a measuring instrument without recourse to any specific physical hardware. A measuring instrument can be described in terms of its functional elements (Fig. 1); and these elements form the bridge between the input to the measurement system and the system output, a quantity that is used to infer the value of the measurand. Most measurement systems fall within the general framework consisting of three functional stages (Holman, 2001):

1. Sensor-transducer stage. Sensor is directly affected by the measurand, while transducer transduces the sensed information to provide an output quantity having a specified relation to the input quantity. Examples of sensors-transducer include thermocouple, strain gauge, manometer, load-cell, etc. There are three basic phenomenon in effect in any sensor operation:

i) The change (or the absolute value) in the measurand causes an equivalent change in the sensor property, e.g., displacement, voltage, resistance, capacitance, inductance, magnetic flux, etc.

ii) The change in the sensor property is converted into a more usable form, e.g., temperature change results in the change in generated voltage by a thermocouple.

iii) The exposure of the sensor to the effects of the measurement environment may lead to some exchange of energy to cause loading effect; e.g., a thermometer when inserted into a cup of tea takes some heat from it to cause a difference between the true value and the indicated value.

2. Signal-conditioning stage. Transduced signal is modified by one or more basic operations, such as amplification, filtering, differentiation, integration, averaging, etc. for further processing, i.e., display, storage or use in feed-back control systems, etc.

3. Output stage. It provides the information sought in a form comprehensible to one of the human senses or to a controller (Beckwith et al., 2007). Output may be analogue or digital, it may be displayed (using LCD or seven-segment display) or saved (using data-loggers) or may be transmitted to a computer or controller (using data-acquisition system) for further use. 


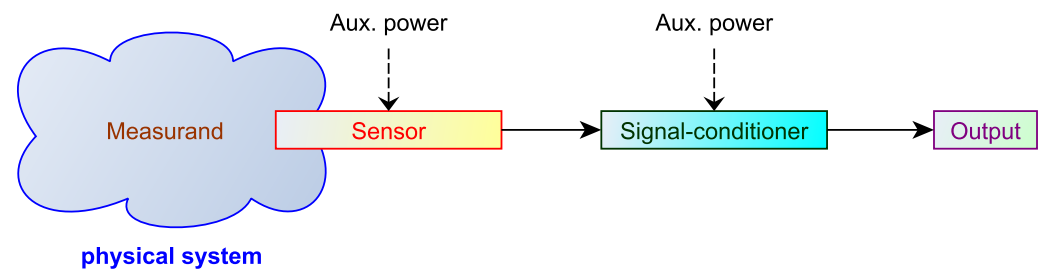

Fig. 1. Functional elements of an instrument or a measurement system.

A glass-bulb mercury thermometer may be analysed to illustrate the above concepts. Here, mercury acts as the sensor whose volume changes with change in its temperature. The transducer is the thermometer-bulb where the change in mercury volume leads to mercury displacement because of the bulb's fixed volume. The stem of the thermometer is the signal-conditioner which physically amplifies the mercury displacement and the graduated scale on the stem offers the required temperature indication. So a pre-established relationship between the input (temperature) and output scale is utilized which is obtained by calibration.

\subsection{Instrument's performance characteristics and calibration}

Measuring instrument transforms the measurand into suitable output that is functionally related to the input and the relationship is established by calibration. Calibration is defined in ISO (2007) as:

operation that, under specified conditions, in a first step, establishes a relation between the quantity values with measurement uncertainties provided by measurement standards and corresponding indications with associated measurement uncertainties and, in a second step, uses this information to establish a relation for obtaining a measurement result from an indication.

Calibration provides the opportunity to check the instrument against a known standard and subsequently to reduce errors in measurements. There is a hierarchy of standards which arranges themselves in order of decreasing accuracy with the primary standards being the most accurate (Doebelin, 2004). Tables 1 and 2, respectively, list hierarchy of standards and errors associated with various levels of temperature standards.

\begin{tabular}{l} 
Primary standard Maintained as absolute unit standard \\
Transfer standard Used to calibrate local standards \\
Local standard Used to calibrate working standards \\
Working standard Used to calibrate local instruments \\
\hline
\end{tabular}

Table 1. Hierarchy of Standards (Figliola \& Beasley, 2011).

\begin{tabular}{lll}
\hline Level & Method & Uncertainty $\left[{ }^{\circ} \mathrm{C}\right]$ \\
\hline Primary & Fixed thermodynamic points & 0 \\
Transfer & Platinum RTD & \pm 0.005 \\
Working & Platinum RTD & \pm 0.05 \\
Local & Thermocouple & \pm 0.5 \\
\hline
\end{tabular}

Table 2. Examples of Temperature Standards (Figliola \& Beasley, 2011).

Calibration is the process of comparison of the output of a measuring system to the values of a range of known inputs and the results may be expressed by a statement, calibration function, 
calibration diagram, calibration curve, or calibration table. In some cases, it may consist of an additive or multiplicative correction of the indication with associated measurement uncertainty. Over the time, it is possible for the indicated values to drift and it makes recalibration necessary at regular intervals. In general, calibration of measuring instruments needs to be traceable to national standardizing laboratory. Hence, traceability is defined by ISO (2007) as:

property of a measurement result whereby the result can be related to a reference through a documented unbroken chain of calibrations, each contributing to the measurement uncertainty.

When the measurand maintains a steady value or slowly varies with time, system performance can be described in terms of static characteristics. Systems dealing with rapidly varying measurand require additional performance parameters termed dynamic characteristics. System performing satisfactorily during static calibration may not provide correct results during dynamic conditions. So relationship between the dynamic input and output must be examined and dynamic performance parameters are required to provide satisfactory results.

\section{Static characteristics and specifications}

When reporting the measurement result of a measurand, it is necessary that some quantitative indication of the quality of the result be given so that those who use it can assess its reliability (ISO, 2008). Without such an indication, measurement results cannot be compared, either among themselves or with reference values given in a specification or standard. ASME Power Test Codes (PTC) 19.1: Test Uncertainty (ASME, 2005) cites the following objectives of uncertainty analysis:

- To facilitate communication regarding measurement and test results;

- To foster an understanding of potential error sources in a measurement system and the effects of those potential error sources on test results;

- To guide the decision-making process for selecting appropriate and cost-effective measurement systems and methodologies;

- To reduce the risk of making erroneous decisions; and

- To document uncertainty for assessing compliance with agreements.

\subsection{Measurement errors and uncertainties}

Every measurement has error, which results in a difference between the measured value and the true value. The difference between the measured value and the true value is the total error. Hence, accuracy is defined as the degree of conformity of an indicated value to a recognized accepted standard, or ideal or true value (ISO, 2007). Since the true value is unknown, total error cannot be known and therefore only its expected values can be estimated.

ASME (2005) quantifies the following two components of total error:

1. Random error, $\epsilon$, is the portion of the measurement error that varies randomly in repeated measurements throughout the conduct of a test (ISO, 2007). Random errors may arise from uncontrolled test conditions and nonrepeatabilities in the measurement system, measurement methods, environmental conditions, data reduction techniques, etc.

2. Systematic error, $\beta$, is the component of measurement error that in replicate measurements remains constant or varies in a particular manner (ISO, 2007). These errors may arise from imperfect calibration corrections, measurement methods, data reduction techniques, etc. 
Random errors are identified and quantified through repeated measurements while trying to keep the conditions constant and by statistical analysis of the results. Systematic errors can be revealed when the conditions are varied, whether deliberately or unintentionally. Figliola \& Beasley (2011) reported the following four methodologies to reduce systematic errors:

1. Calibration, by checking the output(s) for known input(s).

2. Concomitant method, by using different methods of estimating the same thing and comparing the results.

3. Inter-laboratory comparison, by comparing the results of similar measurements.

4. Experience.

Errors in the measurement process are classified into four groups (ASME, 2005; Figliola \& Beasley, 2011) and examples of the error sources are reported in Table 3.

\begin{tabular}{ll}
\hline Error group & Error source \\
\hline 1. Calibration error & Standard or reference value errors \\
& Instrument or system errors \\
& Calibration process errors \\
& Calibration curve fit \\
\hline 2. Loading error & Interaction between the instrument and test media \\
& Interaction between test article and test facility \\
\hline 3. Data-acquisition error & Measurement system operating conditions \\
& Sensor-transducer stage (instrument error) \\
& Signal conditioning stage (instrument error) \\
& Output stage (instrument error) \\
& Process operating conditions \\
& Sensor installation effects \\
& Environmental effects \\
& Spatial variation error \\
& Temporal variation error \\
\hline 4. Data-reduction error & Calibration curve fit \\
& Truncation error \\
\hline
\end{tabular}

Table 3. Error classifications and sources.

It is now widely recognized that, when all of the known or suspected components of error have been evaluated and the appropriate corrections have been applied, there still remains an uncertainty about the correctness of the stated result, i.e., a doubt about the quality of the result of the measurement. The word uncertainty means doubt, and thus in its broadest sense uncertainty of measurement means doubt about the validity of the result of a measurement. According to ISO (2007), uncertainty is defined as:

non-negative parameter characterizing the dispersion of the quantity values being attributed to a measurand, based on the information used.

There are two widely accepted professional documents on uncertainty analysis:

1. ASME Power Test Codes (PTC) 19.1: Test Uncertainty (ASME, 2005), and 
2. ISO/IEC Guide 98:2008 Uncertainty of Measurement - Guide to the Expression of Uncertainty in Measurement (GUM) (ISO, 2008).

These two documents differ in some terminology and how errors are catalogued. For example, ASME (2005) refers to random and systematic error terms to classify errors by how they manifest themselves in the measurement; ISO (2008) refers to Type A and Type B errors. Type A uncertainties have data with which standard deviation can be calculated, while Type B uncertainties do not have data to calculate a standard deviation and must be estimated by other means. These differences are real but the final result of an uncertainty analysis by either method will yield a similar value (Figliola \& Beasley, 2011).

\subsection{Analysis of measurement data}

Any single measurement of a parameter, $x$, is influenced by different elemental random error sources. In successive measurements of the parameter, the values of these elemental random error sources change resulting in the evident random scatter. Measurement scatters common in science and engineering are, in general, described by Normal or Gaussian distribution which predicts that the scatter in the measured data-set will be distributed symmetrically about some central tendency (Fig. 2). If an infinite number of measurements of a parameter were to be taken following the defined test process, the resulting population of measurements could be described statistically in terms of the population mean, $\mu$, and the population standard deviation, $\sigma$; and the true value of $x$ would be $\mu$ (Doebelin, 2004).

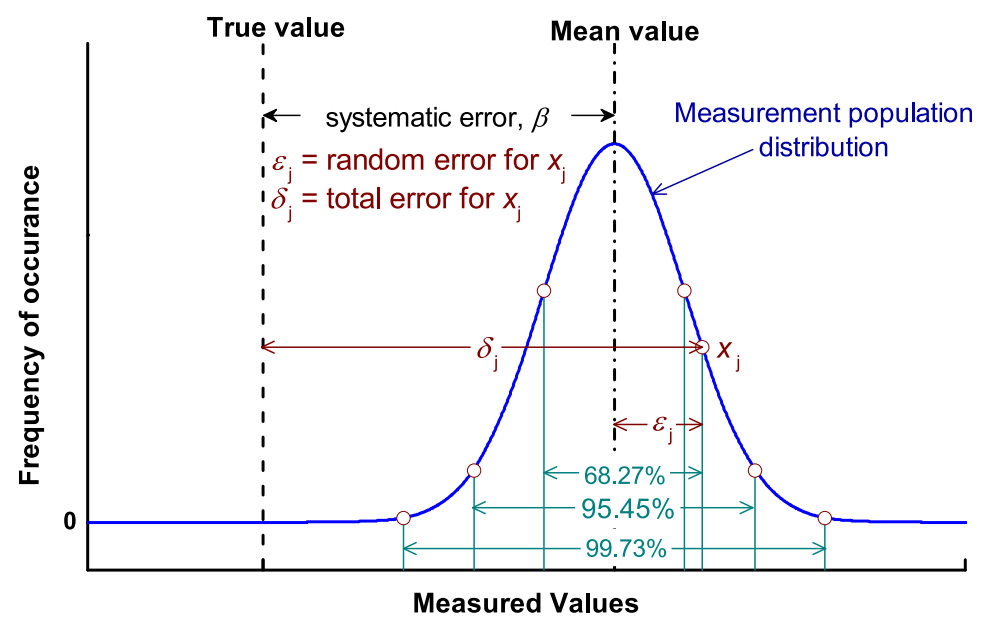

Fig. 2. Illustration of measurement errors.

In practice, only finite-sized data are available. So measured data can only provide an estimate of the true value (Figliola \& Beasley, 2011). If the measured variable is described by discrete data of size $N$, the mean value, $\bar{x}$, of the measured value, $x_{j}$ is given by:

$$
\bar{x}=\frac{1}{N} \sum_{j=1}^{N} x_{j}
$$


, and the standard deviation is given by

$$
S_{x}=\sqrt{\frac{1}{N-1} \sum_{j=1}^{N}\left(x_{j}-\bar{x}\right)^{2}}
$$

In computing the sample average, $\bar{x}$, all $N$ data are independent. However, the standard deviation uses the result of the previous calculation for $\bar{x}$. Hence, number of degrees of freedom is reduced by one and in calculating the standard deviation, $(N-1)$ is used instead of $N$. It may also be noted that, for infinite number of measurements $(N \rightarrow \infty)$ : $\bar{x} \rightarrow \mu$ and $S_{x} \rightarrow \sigma$. For finite data sets, the standard deviation of the mean, $S_{\bar{x}}$, can be estimated from a single finite data set as:

$$
S_{\bar{x}}=\frac{S_{x}}{\sqrt{N}}
$$

For a normal distribution of $x$ about some sample mean value, $\bar{x}$, it can be stated statistically

$$
x_{j}=\bar{x} \pm u_{x}=\bar{x} \pm k(v, \gamma) S_{x}=\bar{x} \pm t(v, \gamma) S_{x} \quad(P \%)
$$

where the $u_{x}$ is the uncertainty interval or precision index associated with the estimate of $x$. The value of $u_{x}$ depends on a constant known as coverage factor that depends on the probability distribution function, the confidence level, $\gamma$ (which is expressed by probability, $P \%$ ) and the amount of data, $N$. For finite data set, $k(v, \gamma)=t(v, \gamma)$. The Student's $t$ value for a given probability, $P \%$ and degrees of freedom in data, $v=N-1$, can be obtained from Table 4, which is a short tabulation of the Student's t distribution. The estimate of the true mean value, $x_{t}$, based on the finite data-set can be estimated (Figliola \& Beasley, 2011) using:

$$
x_{t}=\bar{x} \pm k(v \gamma) S_{\bar{x}} \quad(P \%)
$$

\begin{tabular}{rrrrr}
\hline$v$ & $t_{50}$ & $t_{90}$ & $t_{95}$ & $t_{99}$ \\
\hline 1 & 1.000 & 6.314 & 12.706 & 63.657 \\
2 & 0.816 & 2.920 & 4.303 & 9.925 \\
5 & 0.727 & 2.015 & 2.571 & 4.032 \\
10 & 0.700 & 1.812 & 2.228 & 3.169 \\
20 & 0.687 & 1.725 & 2.086 & 2.845 \\
30 & 0.683 & 1.697 & 2.042 & 2.750 \\
$\infty$ & 0.674 & 1.645 & 1.960 & 2.576 \\
\hline
\end{tabular}

Table 4. Student's t distribution (Figliola \& Beasley, 2011).

The uncertainty interval, $u_{x}$, assumes a set of measured values with only random error present. Furthermore, the set of measured values is assumed to have unbound significant digits and to have been obtained with a measuring system having infinite resolution. When finite resolution exits and truncation of digits occurs, the uncertainty interval may be larger than that predicted by the consideration of the random errors only. The uncertainty interval can never be less that the resolution limits or truncation limits of the measured values.

To illustrate the above concepts, consider the data of Table 5, where 20 values of an arbitrary measurement is presented. 


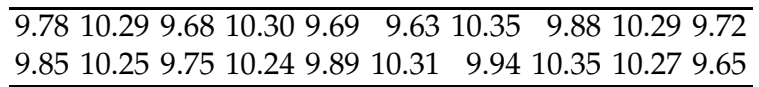

Table 5. Sample of random variable, $x$.

- Sample mean, $\bar{x}=\frac{1}{N} \sum_{j=1}^{N} x_{j}=10.0$

- Standard deviation, $S_{x}=\sqrt{\frac{1}{N-1} \sum_{j=1}^{N}\left(x_{j}-\bar{x}\right)^{2}}=0.28$

- If a $21^{\text {st }}$ data points are to be taken:

$$
\begin{array}{rlr}
x_{j=21}=10.00 \pm(1.729 \times 0.28)=10.00 \pm 0.48 & (90 \%) \\
& =10.00 \pm(2.093 \times 0.28)=10.00 \pm 0.59 \quad(95 \%)
\end{array}
$$

So, there is a $95 \%$ probability that the $21^{\text {st }}$ value will be between 9.41 and 10.59 .

- The true value, $x_{t}$, is estimated by the sample mean value, $\bar{x}$ and the standard deviation of the mean, $S_{\bar{x}}=S_{x} / \sqrt{N}=0.0626$ :

$$
\begin{array}{rlr}
x_{t} & =10.00 \pm 0.11 \quad(90 \%) \\
& =10.00 \pm 0.13 \quad(95 \%)
\end{array}
$$

\subsection{Uncertainty analysis: error propagation}

In calibration experiments, one measures the desired results directly. In nearly all other measurements, results are obtained through functional relationship with measured values. So, it is necessary to compute the uncertainty in the results from the estimates of the uncertainty in the measurements. This computation process is called the propagation of uncertainty.

Consider a result, $R$, which is determined through some functional relationship between independent variables, $x_{1}, x_{2}, \cdots, x_{N}$, defined by

$$
R=R\left(x_{1}, x_{2}, \cdots, x_{N}\right)
$$

where $N$ is the number of independent variables and each variable contains some measure of uncertainty to affect the final result. The best estimate of the true mean value, $R_{t}$, would be stated as:

$$
R_{t}=\bar{R} \pm u_{R} \quad(P \%)
$$

where the sample mean, $\bar{R}$ is found from

$$
\bar{R}=R\left(\bar{x}_{1}, \bar{x}_{2}, \cdots, \bar{x}_{N}\right)
$$

and the uncertainty in $\bar{R}$ is found from

$$
u_{R}=\sqrt{\left(\frac{\partial R}{\partial x_{1}} u_{1}\right)^{2}+\left(\frac{\partial R}{\partial x_{2}} u_{2}\right)^{2}+\cdots+\left(\frac{\partial R}{\partial x_{N}} u_{N}\right)^{2}}
$$

where, $u_{1}, u_{2}, \cdots, u_{N}$ are the uncertainties associated with $x_{1}, x_{2}, \cdots, x_{N}$.

To illustrate the concepts presented in the present section, let us consider the calculation of electrical power, $P$ from

$$
P=V I
$$


, where, voltage, $V$ and current, $I$ are measured as

$$
\begin{gathered}
V=100 \mathrm{~V} \pm 5 \mathrm{~V} \\
I=10 \mathrm{~A} \pm 0.1 \mathrm{~A}
\end{gathered}
$$

Hence, the nominal value of power is $1000 \mathrm{~W}$. By taking the worst possible variations in voltage and current, we would calculate

$$
\begin{aligned}
& P_{\text {max }}=(100+5)(10+0.1)=1060.5 \mathrm{~W} \\
& P_{\text {min }}=(100-5)(10-0.1)=940.5 \mathrm{~W}
\end{aligned}
$$

Thus, using the simple calculations, the uncertainty in the power is $+6.05 \%,-5.95 \%$. However, it is quite unlikely that the power would be in error by these amount because the variation of voltage reading would probably not be corresponding with the current reading. Hence, it is quite unlikely these two independent parameters would achieve maximum or minimum values simultaneously. Hence, using Eq. 9:

$$
\begin{array}{cc}
\frac{\partial R}{\partial x_{1}}=\frac{\partial P}{\partial V}=I=10 \mathrm{~A} & u_{1}=u_{V}=5 \mathrm{~V} \\
\frac{\partial R}{\partial x_{2}}=\frac{\partial P}{\partial I}=V=100 \mathrm{~V} & u_{2}=u_{I}=0.1 \mathrm{~A} \\
u_{R}=\sqrt{(10 \times 5)^{2}+(100 \times 0.1)^{2}}=\sqrt{2500+100}=51.0 \mathrm{~W}=5.1 \%
\end{array}
$$

Hence, estimated power, $P=1000 \mathrm{~W} \pm 5.1 \%$.

If we ignore the uncertainty contribution due to the current reading $\left(u_{I} \rightarrow 0\right)$, the uncertainty in power measurement would be $5 \%$. Hence, very little is gained by trying to reduce the 'small' uncertainties. Any improvement in result should be achieved by improving the instrumentation or technique connected with relatively large uncertainties.

\section{Specification of dynamic systems and response}

A measuring instrument requires a certain amount of time to achieve complete response. In case of a dynamic measurement, actual response undergoes some attenuation, delay and distortion. A measurement system should be capable of yielding the true value(s) from the time varying input value(s). Study of dynamic characteristics of such system requires considerable analyses and the responses are conveniently stated in terms of step response, harmonic response and frequency response of the instrument.

\subsection{Modelling of measuring instruments}

The response of a measurement system, i.e., output, $x(t)$, when subjected to an input forcing function, $f(t)$, may be expressed by a linear ordinary differential equation with constant coefficients of the form (Doebelin, 2004):

$$
a_{n} \frac{d^{n} x}{d t^{n}}+a_{n-1} \frac{d^{n-1} x}{d t^{n-1}}+\cdots+\underbrace{a_{2} \frac{d^{2} x}{d t^{2}}+\underbrace{a_{1} \frac{d x}{d t}+\overbrace{a_{0} x=f(t)}^{0^{\text {th }} \text { order }}}_{1^{\text {st }} \text { order }}}_{2^{\text {nd }} \text { order }}
$$


$f(t) \triangleq$ Input quantity imposed on the system,

where, $x(t) \triangleq$ Output or the response of the system,

$a^{\prime} \mathrm{s} \triangleq$ Physical system parameters, assumed constants.

Hence, the order of the system is designated by the order of the differential equation. Once the governing equation of the instrument is established, its response can be obtained if the input (measurand) is a known function of time. Dynamic response of a system can be estimated by solving Eq. 10 with proper initial and boundary conditions. While the general model of Eq. 10 is adequate for handling any linear measurement system, certain special cases (e.g., zero-, first- and second-order systems) occur so frequently in practice that warrant special attention. Furthermore, complex systems may profitably be considered in terms of the combinations of simple cases and the response can be inferred from the observations made from these cases.

\subsubsection{Zero-order instrument}

The simplest case of Eq. 10 occurs if all the values of $a^{\prime}$ s other than $a_{0}$ are assumed to be zero, and Eq. 10 is then transformed into a simple algebraic equation:

$$
a_{0} x=f(t): \Longrightarrow x(t)=\mathbb{k} f(t)
$$

The constant, $\mathbb{k} \triangleq 1 / a_{0}$ is called the static sensitivity of the system, and it represents the scaling between the input and the output. For any-order system, static sensitivity always has the same physical interpretation, i.e., the amount of output per unit input when the input is static and under such condition all the derivative terms of Eq. 10 are zero.

A practical example of a zero-order instrument is a displacement measuring potentiometer where a strip of resistance material is excited with a voltage, $V_{s}$, and provided with a sliding contact responding to displacement. If the resistance is linearly distributed along the length, $L$, the output voltage, $e_{o}(t)$, may be written as a function of displacement, $l(t)$, as:

$$
e_{0}(t)=\frac{V_{s}}{L} l(t)=\mathbb{k} l(t)
$$

where, $\mathbb{k} \triangleq V_{s} / L$ (Volts per unit length).

\subsubsection{First-order instrument}

If in Eq. 10, all $a^{\prime}$ s other than $a_{1}$ and $a_{o}$ are taken as zero, we get:

$$
a_{1} \frac{d x}{d t}+a_{0} x=f(t): \Longrightarrow \tau \frac{d x}{d t}+x=\mathbb{k} f(t)
$$

where, $\begin{aligned} & \mathbb{k} \triangleq 1 / a_{0} \triangleq \text { static sensitivity, } \\ & \tau \triangleq a_{1} / a_{0} \triangleq \text { time-constant. }\end{aligned}$

The time constant has the dimension of time, while the static sensitivity has the dimension of output divided by input. When time constant of a system is very small, the effect of the derivative term in Eq. 13 becomes negligible and the governing equation approaches to that of a zero-order system. It is experimentally observed that an instrument with small value of the time-constant is associated with good dynamic response. 
A practical example of a first-order instrument is a mercury-in-glass thermometer. If a thermometer, initially at room temperature, is inserted into a hot fluid at temperature, $T_{\infty}$, then the convective heat gain from the hot fluid will result in the increase of internal energy of mercury at the same rate. The thermometer takes a while to give the correct reading, and the temperature reading, $T(t)$, can be obtained using the following energy-balance equation:

$$
\dot{Q}_{i n}=h A\left[T_{\infty}-T(t)\right]=m C \frac{d T(t)}{d t}: \Longrightarrow \tau \frac{d T(t)}{d t}+T(t)=T_{\infty}
$$

Hence, $\tau \triangleq m C / h A$ and $\mathbb{k}=1$; and $h$ is the convective heat transfer coefficient, $A$ is the surface area over which heat is transferred, $m$ is the mass of mercury, and $C$ is its specific heat.

\subsubsection{Second-order instrument}

A second-order instrument is one that follows the equation:

$$
a_{2} \frac{d^{2} x}{d t^{2}}+a_{1} \frac{d x}{d t}+a_{0} x=f(t): \Longrightarrow \frac{1}{\omega_{n}^{2}} \frac{d^{2} x}{d t^{2}}+2 \frac{\zeta}{\omega_{n}} \frac{d x}{d t}+x=\mathbb{k} f(t)
$$

$\mathbb{k} \triangleq 1 / a_{0} \triangleq$ static sensitivity,

where, $\omega_{n} \triangleq \sqrt{\frac{a_{0}}{a_{2}}} \triangleq$ undamped natural frequency,

$\zeta \triangleq \frac{a_{1}}{2 \sqrt{a_{0} a_{2}}} \triangleq$ dimensionless damping ratio.

A practical example of a second-order instrument is a weight measuring spring balance as shown in Fig. 3 . Hence, $k$ is the spring constant of the ideal spring and $b$ is the friction constant where damper friction force is linearly proportional to the velocity of mass, $\dot{x}(t)$. Summing the forces acting on the mass and utilizing the Newton's second law of motion yields,

$$
f(t)-k x-b \frac{d x}{d t}=m \frac{d^{2} x}{d t^{2}}: \Longrightarrow \frac{1}{\omega_{n}^{2}} \frac{d^{2} x}{d t^{2}}+2 \frac{\zeta}{\omega_{n}} \frac{d x}{d t}+x=\mathbb{k} f(t)
$$

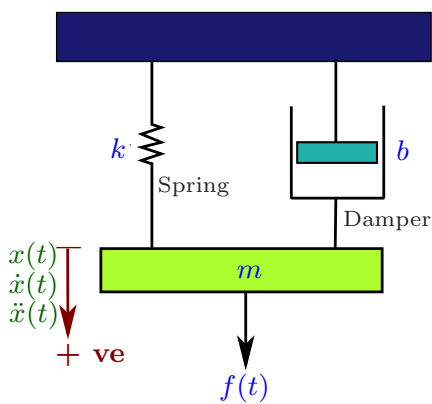

(a)

$$
\begin{aligned}
k & \equiv \text { spring constant } & f(t) & \equiv \text { forcing function } \\
b & \equiv \text { damping constant } & m & \equiv \text { mass }
\end{aligned}
$$

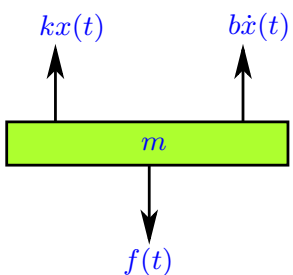

(b)

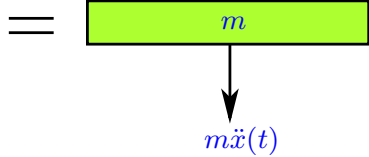

Fig. 3. (a) Spring-mass-damper system, (b) Free-body diagram.

Final form of Eq. 15, could represent, among other things, an electrical resistor-inductor-capacitor (RLC) circuit. The variables of the electric circuit behave exactly as the analogous variables of the equivalent mechanical system. The spring-mass-damper system and analogous RLC circuit are illustrated in Fig. 4. Both of these systems share the same governing equation and therefore have analogous response when subjected to input forcing. 


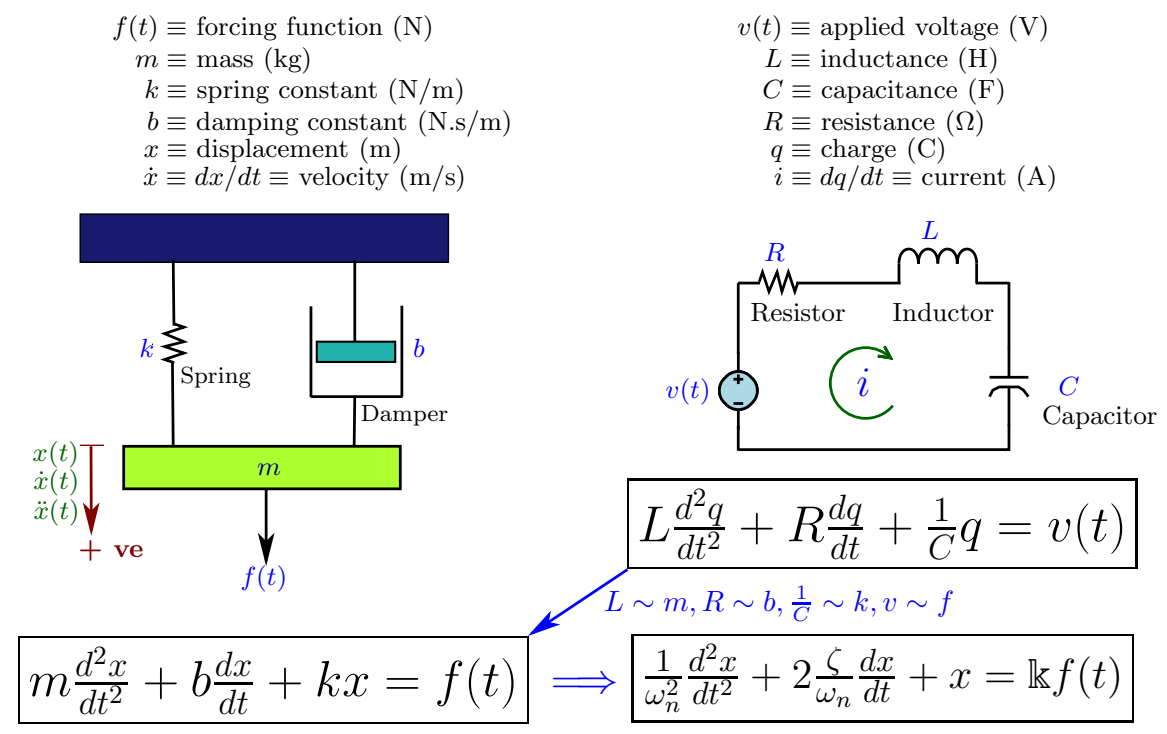

Fig. 4. Spring-mass-damper system and analogous RLC circuit.

\subsection{Modelling of response of measuring instruments}

Once the governing equation of a system is established, its dynamic response to forcing element can be obtained if the forcing can be represented as a function of time. The fundamental difficulty in this approach lies in the fact that the quantities to be measured usually do not follow some simple mathematical functions. However, it is fruitful to study a system's response to common forcing inputs, e.g., step and harmonic inputs. These forcing functions have been found to be very useful in the theoretical and experimental analyses of measurement system's response. Mathematically, the step function of magnitude, $A$, can be expressed as:

$$
f(t)= \begin{cases}0 & \text { at } t=0 \\ A & \text { for } t>0\end{cases}
$$

, and the harmonic function of circular frequency, $\omega$, can be expressed as:

$$
f(t)= \begin{cases}0 & \text { at } t=0 \\ A \sin \omega t & \text { for } t>0\end{cases}
$$

Graphical representation of these two functions are shown in Fig. 5.

\subsubsection{Response of zero-order instruments}

The response of a zero-order instrument is governed by a simple algebraic equation (Eq. 11). So the output, $x(t)$, follows the input, $f(t)$, perfectly with no distortion or time lag. Hence, zero-order instrument represents ideal dynamic performance, and is thus a standard against which less perfect or dynamic instruments are compared. Typical responses of a zero-order instrument are shown in Fig. 6. 


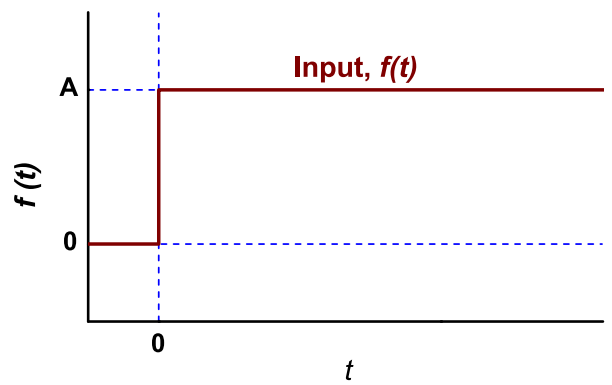

(a) Step input

Fig. 5. Step and harmonic inputs.

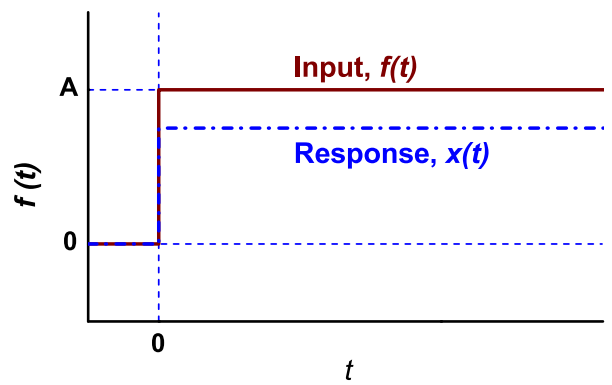

(a) Step input

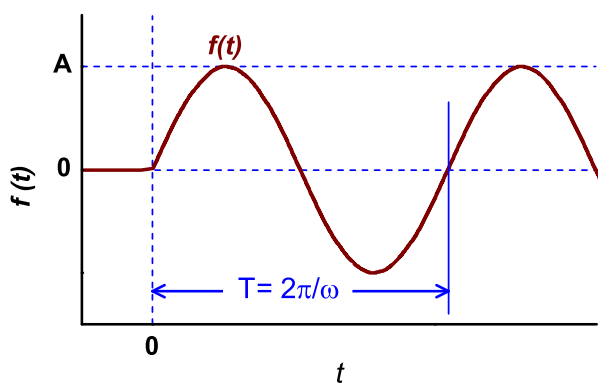

(b) Harmonic input

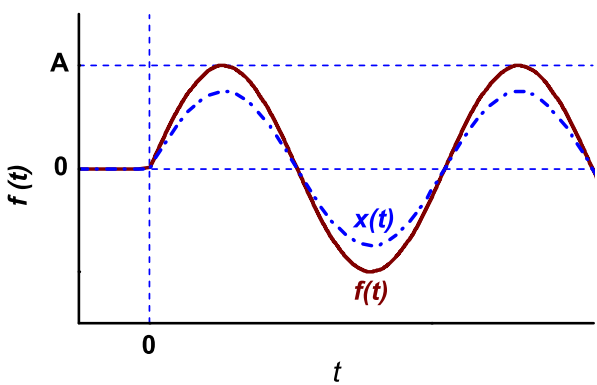

(b) Harmonic input

Fig. 6. Zero-order instrument's response for step and harmonic inputs (for $\mathbb{k}=0.75$ ).

Zero-order system concept is used to analyse real system's response in static calibration. When dynamic signals are involved, higher order differential equations are required to estimate the dynamic response as most of the real systems possess inertial/storage capability and are subjected to some viscous resistance and dissipation (Figliola \& Beasley, 2011).

\subsubsection{Response of first-order instruments}

Response for Step Input: If the governing equation for first-order system (Eq. 13) is solved for a step input function and with initial condition $\left.x\right|_{t=0}=x_{0}$, the solution is:

$$
x(t)=\underbrace{\left(x_{0}-A \mathbb{k}\right) \exp (-t / \tau)}_{\text {transient response }}+\underbrace{A \mathbb{k}}_{\text {steady-state response }}
$$

For large values of time, $x(t \rightarrow \infty)=A \mathbb{k} \triangleq x_{\infty}$. Hence, $x_{\infty} \triangleq$ steady-state response of the system. Equation 19 can be rearranged to give non-dimensional response, $M(t)$, as:

$$
M(t)=\frac{x(t)-x_{o}}{x_{\infty}-x_{0}}=1.0-\exp (-t / \tau)
$$

, where, $M(t)$ is the ratio between output and input amplitudes. Non-dimensional Eq. 20 is valid for all first-order system's response to step-input forcing; and plot of Eq. 20 is shown in 
Fig. 7. It is observed that, the response approaches the steady-state value monotonically and the response reaches $63.2 \%$ of its steady-state value when $t=\tau$. When elapsed time is $2 \tau$, $3 \tau$, and $4 \tau$, the response is $86.5 \%, 95 \%$ and $98 \%$, respectively. It is also seen that, the slope of the response curve (Fig. 7) at the origin is 1.0, i.e., if the initial response rate were maintained, the response would be completed in one time constant. However, the final value (steady-state response) is achieved only after infinite time (Fig. 7). In practice two response specifications are used to describe the first-order system response:

1. Settling time, $t_{s}$. It is defined as the time required for the system to reach and stay within the $2 \%$ of the final value. Hence, for first-order system, $t_{s} \simeq 4 \tau$.

2. Rise time, $t_{r}$. It is defined as the time required for the response to go from a small percentage $(10 \%)$ to a large percentage $(90 \%)$ of the step input. Hence, for first-order system, $t_{r} \simeq 2.2 \tau$.

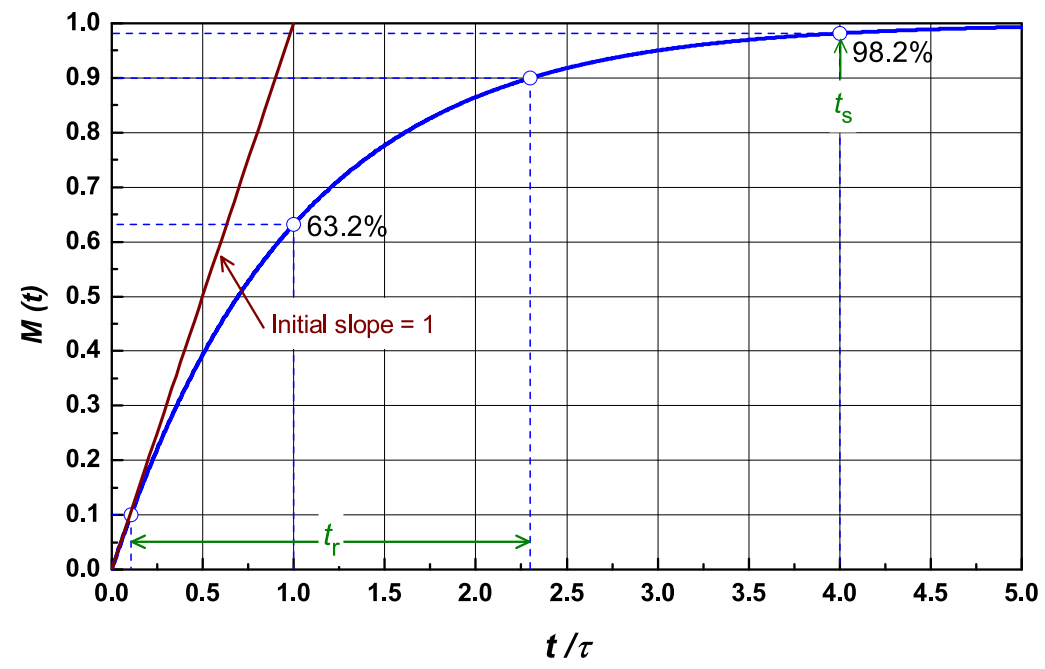

Fig. 7. First-order system response to a unit step-input.

Response for Harmonic Input: If the governing equation for first-order system (Eq. 13) is solved for harmonic input and $\left.x\right|_{t=0}=0$, the solution is:

$$
\frac{x(t)}{A \mathbb{k}}=\underbrace{\frac{\omega \tau}{1+(\omega \tau)^{2}} \exp (-t / \tau)}_{\text {transient response }}+\underbrace{\frac{1}{\sqrt{1+(\omega \tau)^{2}}} \sin (\omega t+\phi)}_{\text {steady-state response }}
$$

where, $\phi \triangleq \tan ^{-1}(-\omega \tau) \triangleq$ phase lag. Hence, time delay, $\Delta t$, is related to phase lag as:

$$
\Delta t=\frac{\phi}{\omega}
$$

For $\omega \tau>>1$, response is attenuated and time/phase is lagged from input, and for $\omega \tau<<1$, the transient response becomes very small and response follows the input with small attenuation and time/phase lag. Ideal response (without attenuation and phase lag) is obtained when the system time constant is significantly smaller than the forcing element 
period, $T$. As $t \rightarrow \infty$, the first term on the right side of Eq. 21 vanishes and leaves only the steady-state solution:

$$
\left.x(t)\right|_{s}=\frac{A \mathbb{k}}{\sqrt{1+(\omega \tau)^{2}}} \sin (\omega t+\phi)=\frac{A \mathbb{k}}{\sqrt{1+(\omega \tau)^{2}}} \sin \omega(t+\Delta t)=f(t) \times G_{a} \angle \phi
$$

Hence, $G_{a} \triangleq \mathbb{k} / \sqrt{1+(\omega \tau)^{2}} \triangleq$ steady-state gain. Equation 23 indicates that the attenuated steady-state response is also a sine wave with a frequency equal to the input signal frequency, $\omega$, and it lags behind the input by phase angle, $\phi$.

To illustrate the above concepts, the response of a thermocouple subjected to a harmonic temperature variation is plotted in Figs. 8 and 9; and summary results are also reported in Table. 6. Initial transient response is clearly visible and steady-state response is achieved only after the expiration of $4 \tau$ time. The response is of same frequency but attenuated in magnitude and lags behinds the input signal. However, once the steady-state is achieved, knowing the values of the gain, $G_{a}$, and phase lag, $\phi$, it is possible to estimate the correct input value, and an example is also shown in Fig. 8. The effect of time constants on dynamic response is illustrated in Fig. 9 where response from three thermocouple systems with time constants of 1 , 5 and $50 \mathrm{~s}$ are plotted. Figure 9 provides a meaningful insight into the effects of time constant as it shows that systems with small time constants follow the input with less attenuation and time delay, and vice versa. However, when average value of a measurand is desired (e.g., in chemical reactant mixing chamber), system with higher time constants are suitable and near average result is observed as shown in Fig. 9 for a thermocouple with $\tau=50 \mathrm{~s}$.

\begin{tabular}{c|ccccc}
\hline Unit & $\tau[\mathrm{s}]$ & $\tau / T$ & $\phi[\mathrm{deg}]$ & $\Delta t[\mathrm{~s}]$ & $G_{a}$ \\
\hline 01 & 01 & 0.04 & -14.0 & -0.98 & 0.97 \\
02 & 05 & 0.2 & -51.3 & -3.58 & 0.62 \\
03 & 50 & 2.0 & -85.4 & -5.96 & 0.08 \\
\hline
\end{tabular}

Table 6. Thermocouple's response to harmonic temperature variation.

\subsubsection{Response of second-order instruments}

Response for Step Input: For a second-order system subjected to a step input, the solution (response) is readily available (Dunn, 2010) and may be written as:

$$
\frac{x(t)}{\mathbb{k} A}= \begin{cases}1-\cos \left(\omega_{n} t\right) & \text { for } \zeta=0 \\ 1-\frac{1}{\sqrt{1-\zeta^{2}}} \exp \left(-\zeta \omega_{n} t\right) \sin \left[\omega_{n} t \sqrt{1-\zeta^{2}}+\cos ^{-1}(-\zeta)\right] & \text { for } 0<\zeta<1 \\ 1-\left(1+\omega_{n} t\right) \exp \left(-\omega_{n} t\right) & \text { for } \zeta=1 \\ 1-\exp \left(-\zeta \omega_{n} t\right)\left[\cosh \left(\omega_{n} t \sqrt{\zeta^{2}-1}\right)+\frac{\zeta}{\sqrt{\zeta^{2}-1}} \sinh \left(\omega_{n} t \sqrt{\zeta^{2}-1}\right)\right] & \text { for } \zeta>1\end{cases}
$$

The nature of the response of the second-order system for a step input depends on the value of the damping ratio, $\zeta$, as depicted in Fig. 10, and four types of response may be identified:

1. Harmonic oscillation $(\zeta=0)$. Response oscillates at the natural frequency, $\omega_{n}$, and the oscillations are undamped. 


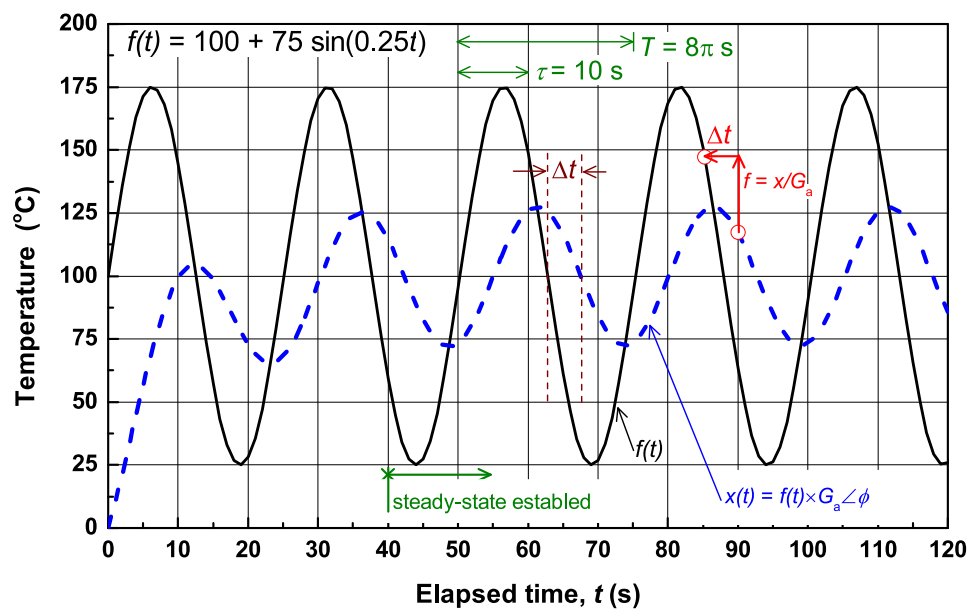

Fig. 8. Response of thermocouple for harmonic temperature input.

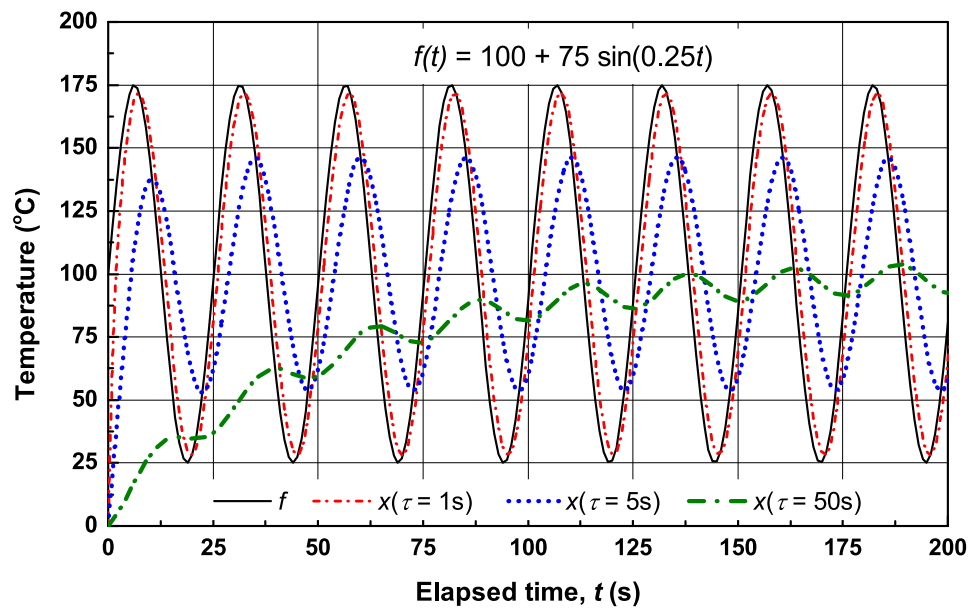

Fig. 9. Effect of time constant on thermocouple's response to harmonic temperature input.

2. Underdamped response $(0<\zeta<1)$. Response overshoots the steady-state value initially, and then eventually decays to the steady-state value. The smaller the value of $\zeta$, the larger the overshoot.

3. Critically damped response $(\zeta=1)$. An exponential rise in response occurs to approach the steady-state value without any overshoot, and the response is as fast as possible without overshoot.

4. Overdamped response $(\zeta>1)$. Response approaches the steady-state value without overshoot, but at a slower rate. Hence, excessive time is required to complete a measurement and therefore frequency at which the measurement is possible is limited. So, little attention is focused on the overdamped systems for dynamic measurements. 


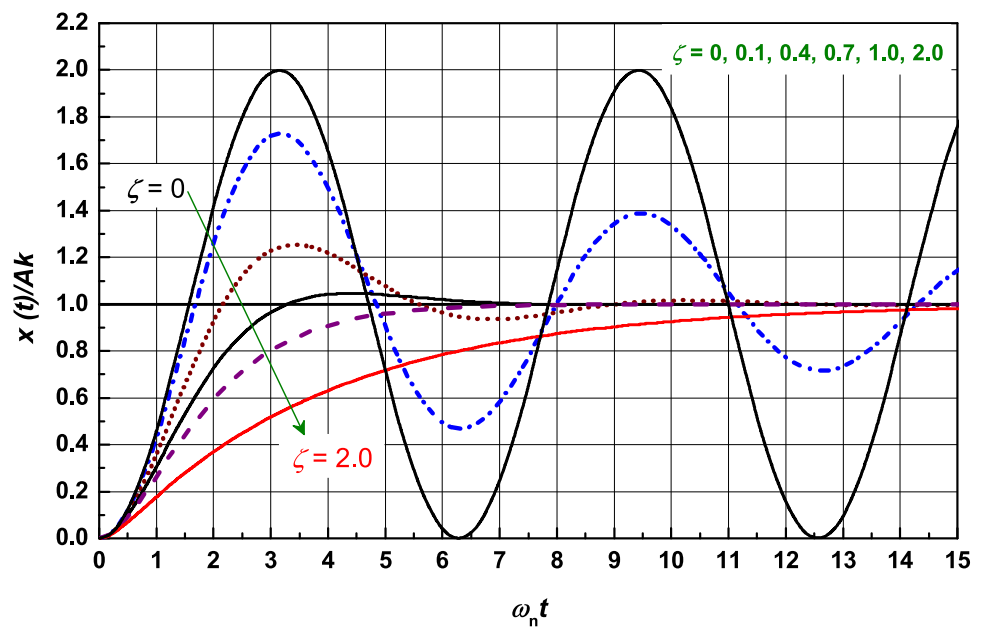

Fig. 10. Transient response of a second-order system for a step-input at $t=0$.

For underdamped system, as $\zeta$ decreases, response becomes increasingly oscillatory. The transient response oscillates about the steady-value and occurs with a period, $T_{d}$, given by:

$$
T_{d} \triangleq \frac{2 \pi}{\omega_{d}} \quad: \quad \omega_{d} \triangleq \omega_{n} \sqrt{1-\zeta^{2}}
$$

, where, $\omega_{d} \triangleq$ ringing frequency. Standard performance parameters in terms of step response is shown in Fig. 11. It is observed that, the duration of the transient response is controlled by $\zeta \omega_{n}$. In fact, its influence is equivalent to that of a time constant in a first-order system, such that we could define a second-order time constant as $\tau_{e} \triangleq 1 / \zeta \omega_{n}$. The system settles to steady state value, $x_{\infty}=\mathbb{k} A$, more quickly when it is designed with large $\zeta \omega_{n}$, i.e., small $\tau_{e}$. Nevertheless, for all systems with $\zeta>0$, the response will eventually indicate the steady value as $t \rightarrow \infty$ (Figliola \& Beasley, 2011). Like a first-order system, the swiftness of the response may be described by settle time, $t_{s}$ and rise time, $t_{r}$. For second-order system, $t_{s} \simeq 4 \tau_{e}$ and $t_{r} \omega_{n} \cong 2.16 \zeta+0.60$ for $0.3 \leqq \zeta \leqq 0.8$ (Dorf \& Bishop, 1998).

Equation 24 also reveals that for second-order system, whenever $\omega_{n}$ appears, it appears as the product of $\omega_{n} t$. This means that, if we say, double $\omega_{n}$, the same range of the response will occur in exactly one half the time. Thus $\omega_{n}$ is a direct and proportional indicator of response speed. For a fixed value of $\omega_{n}$, the speed of response is determined by $\zeta$ (Doebelin, 1998).

\subsubsection{Response in time-domain and postscript}

The response of a zero-order system is an ideal one and response follows the input without attenuation and phase lag. In practice, no measurement system is ideal and therefore does not possess zero-order characteristics. First-order system follows the input signal with some attenuation and phase lag, but no oscillations are present. Second-order system responses are further complicated as in addition to output attenuation and phase lag, it may exhibit oscillation. The total response of a non-zero-order dynamic system due to a harmonic input is the sum of transient response, which is independent of the frequency of the input, and a 


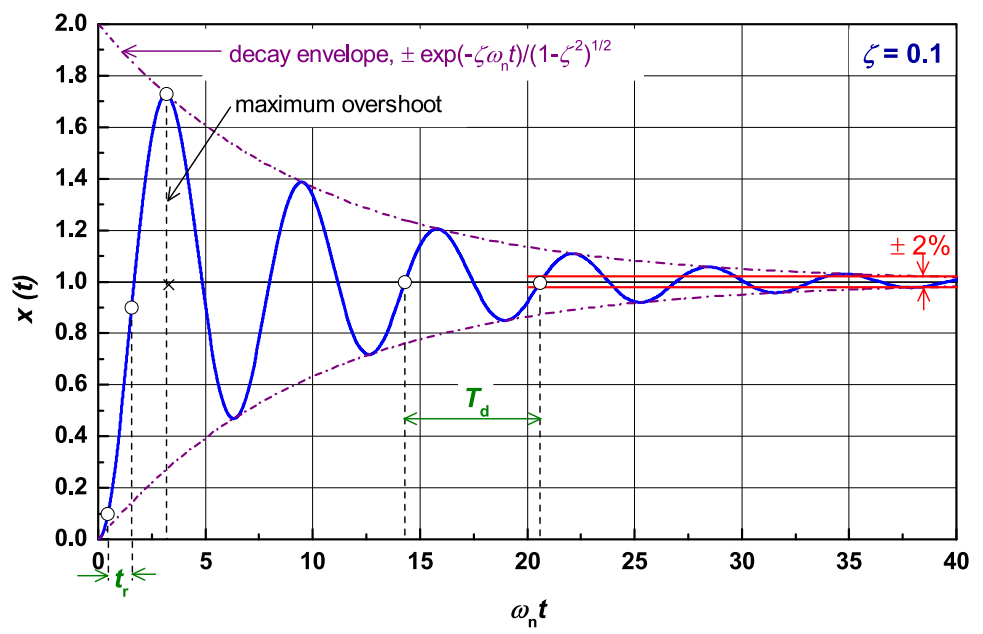

Fig. 11. Second-order underdamped response specifications.

response which depends on the frequency of the input (Dorf \& Bishop, 1998). For a stable system, the transient response decays exponentially and eventually becomes insignificant compared to the response that is specific to the input. When this occurs, the system has reached the steady-state response (Kelly, 2003). Time-domain analysis is often complicated because of the initial transient-response. However, in a measurement system, we are interested in the steady-state response where the system responds to the input without being affected by the transient effect of the system itself.

\subsection{Frequency response of instruments}

A very practical and convenient alternative to time-domain modelling to capture the dynamic response of linear system is through its frequency response which is defined as the steady-state response of the system to a sinusoidal input. The sinusoidal is a unique input signal, and the resultant output signal for a linear system as well as signal throughout the system, is sinusoidal in the steady-state; it differs from the input wave-form only in amplitude and phase angle (Dorf \& Bishop, 1998). Moreover, almost all types of functions can be described through Fourier analysis in terms of the sums of sine and cosine functions. So, if a linear system's response for sinusoidal input is determined, then its response to more complicated input can be described by linearly superimposing the outputs determined for each of the sinusoidal-input that were identified by Fourier analysis. In frequency response analysis, sinusoidal input signal is varied over a range of frequencies, and for each frequency, there is a gain and phase angle that give the characteristic response at that frequency. The results are plotted in a pair of graphs known as Bode diagram which consists of two plots:

1. Logarithmic gain, $L(\omega) \triangleq 20 \log _{10} G_{a}(\omega)$ vs. $\log _{10}(\omega)$, and

2. Phase angle, $\phi(\omega)$ vs. $\log _{10}(\omega)$

The vertical scale of the amplitude Bode diagram is in decibels $(\mathrm{dB})$, where a non-dimensional frequency parameter such as frequency ratio, $\left(\omega / \omega_{n}\right)$, is often used on the horizontal axis. 
Laplace transformation (LT) is the key to frequency-domain modelling. It is a mathematical tool to transform linear differential equations into an easier-to-manipulate algebraic form. In frequency domain, the differential equations are easily solved and the solutions are converted back into time-domain to provide system response (Fig. 12). Some of the common Laplace transform (LT) pairs are reported in Table 7.

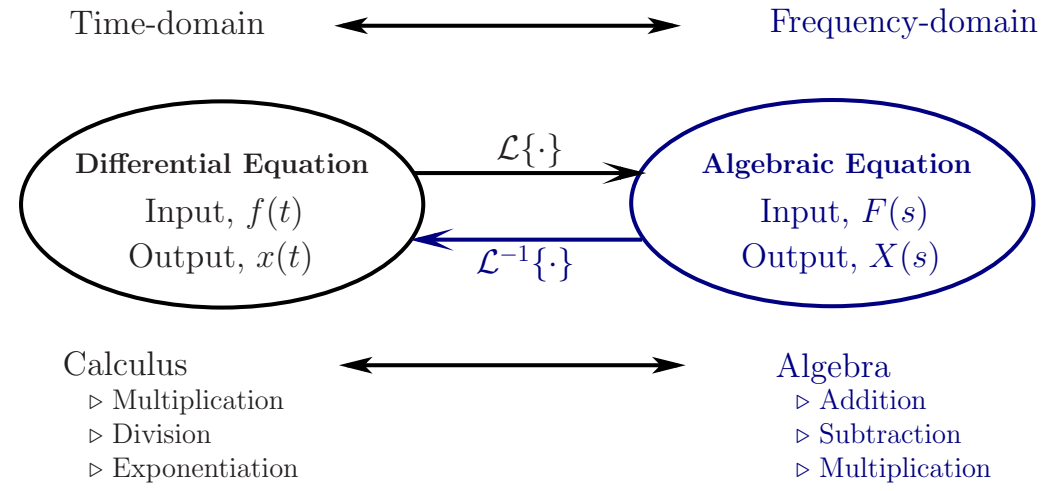

Fig. 12. Laplace transform (LT) takes a function from time-domain to frequency-domain. Inverse LT takes a function from frequency-domain to time-domain.

\begin{tabular}{ll}
\hline$f(t)$ & $F(s)$ \\
\hline$\delta(t)$, unit impulse & 1 \\
Step function, $A$ & $A / s$ \\
$A t ; A t^{n}$ & $\frac{A}{s^{2}} ; \frac{A n}{s^{n+1}}$ \\
$A e^{-a t}$ & $\frac{A+a}{s+a}$ \\
$A \sin (\omega t)$ & $\frac{A \omega}{s^{2}+\omega^{2}}$ \\
$\cos (\omega t)$ & $\frac{s}{s^{2}+\omega^{2}}$ \\
$f^{\prime}(t)$ & $s F(s)-f(0)$ \\
$f^{\prime \prime}(t)$ & $s^{2} F(s)-s f(0)-f^{\prime}(0)$ \\
$\frac{\omega_{n}}{\sqrt{1-\zeta^{2}}} e^{-\zeta \omega_{n} t} \sin \omega_{n} \sqrt{1-\zeta^{2}} t, \zeta<1 \frac{\omega_{n}^{2}}{s^{2}+2 \zeta \omega_{n} s+\omega_{n}^{2}}$ \\
$c_{1} f_{1}(t)+c_{2} f_{2}(t)$ & $c_{1} F_{1}(s)+c_{2} F_{2}(s)$ \\
\hline
\end{tabular}

Table 7. Laplace transform (LT) pairs of some common functions.

\subsubsection{Laplace transform (LT) and Transfer function (TF) methods}

Transfer function (TF) is widely used in frequency-domain analysis and it establishes the size and timing relationship between the output and the input. Transfer function of a linear system, $G(s)$, is defined as the ratio of the Laplace transform (LT) of the output variable, $X(s) \triangleq \mathcal{L}\{x(t)\}$, to the LT of the input variable, $F(s) \triangleq \mathcal{L}\{f(t)\}$, with all the initial conditions are assumed to be zero. Hence,

$$
G(s) \triangleq \frac{X(s)}{F(s)}
$$


The Laplace operator, $s \triangleq \sigma+j \omega$, is a complex variable. Hence, $j \equiv \sqrt{-1}$, and $\omega$ translates into a sinusoid in time-domain, $\sigma$ translates into an exponential term, $\exp (\sigma t)$. For steady-state sinusoidal input, $\sigma=0$, and system response can be evaluated by setting $s=j \omega$. So,

$$
F(s) \longrightarrow G(s) \longrightarrow X(s) \quad: \Longrightarrow \quad x(t)=f(t) \times G_{a} \angle \phi
$$

Hence, $\begin{array}{ll}G(j \omega) & \left.\triangleq G(s)\right|_{s=j \omega} \triangleq \text { steady-state transfer function, } \\ G_{a}(\omega) & \triangleq|G(j \omega)| \triangleq \text { gain frequency response, } \\ \phi(\omega) & \triangleq \angle G(j \omega) \triangleq \text { phase frequency response, } \\ G_{a}(\omega) \angle \phi(\omega) \triangleq G_{a} \angle \phi & \triangleq \text { frequency response. }\end{array}$

For example, transfer function of a first-order system is evaluated below:

- time-domain equation: $\tau \frac{d x}{d t}+x=\mathbb{k} f(t)$

- s-domain equation: $\tau s X(s)+X(s)=\mathbb{k} F(s)$

$$
-F(s) \longrightarrow G(s)=\frac{\mathbb{k}}{1+s \tau} \rightarrow X(s)
$$

- $G_{a}=|G(s \Leftarrow j \omega)|=\left|\frac{\mathbb{k}}{1+j \omega \tau}\right|=\frac{\mathbb{k}}{\sqrt{1+(\omega \tau)^{2}}}$

- $\phi=\angle G(j \omega)=\tan ^{-1}(-\omega \tau)$

- For input, $f(t)=A \sin (\omega t)$, steady-state response, $x(t)=\frac{\mathbb{k} A}{\sqrt{1+(\omega \tau)^{2}}} \sin (\omega t+\phi)$.

Expressions of $G(s), G_{a}$ and $\phi$ for zero-, first- and second-order systems are reported in Table 8 .

\begin{tabular}{l|ccc}
\hline & Zero-order & First-order & Second-order \\
\hline Differential Equation & $x(t)=\mathbb{k} f(t)$ & $\tau \frac{d x}{d t}+x=\mathbb{k} f(t)$ & $\frac{1}{\omega_{n}^{2}} \frac{d^{2} x}{d t^{2}}+2 \frac{\zeta}{\omega_{n}} \frac{d x}{d t}+x=\mathbb{k} f(t)$ \\
Transfer function, $G(s)$ & $\mathbb{k}$ & $\frac{\mathbb{k}}{\tau s+1}$ & $\frac{\frac{1}{\omega_{n}^{2}} s^{2}+2 \frac{\zeta}{\omega_{n}} s+1}{\sqrt{1+(\omega \tau)^{2}}}$ \\
Gain, $G_{a}$ & $\mathbb{k}$ & $\frac{\mathbb{k}}{\sqrt{\left[1-\left(\frac{\omega}{\omega_{n}}\right)^{2}\right]^{2}+4 \zeta^{2}\left(\frac{\omega}{\omega_{n}}\right)^{2}}}$ \\
Phase angle, $\phi$ & 0 & $\tan ^{-1}(-\omega \tau)$ & $\tan ^{-1}\left[-\frac{2 \zeta\left(\frac{\omega}{\omega_{n}}\right)}{\left[1-\left(\frac{\omega}{\omega_{n}}\right)^{2}\right]}\right]$ \\
\hline
\end{tabular}

Table 8. Transfer function, gain and phase angle of zero-, first- and second-order systems.

\subsubsection{Bode-diagram and selection of instrumentation parameters}

Ideal system response of a zero-order system is shown in Fig. 13, where both the values of $L(\omega)$ and $\phi(\omega)$ are zero. In case of a first-order system response, as shown in Fig. 13, when the measurement system responds with values of $L(\omega) \simeq 0$, system transfers all or nearly all of the input signal amplitude to the output and with very little time delay. At larger values of $\omega \tau$, measurement system will essentially filter out frequency information of the input by responding with smaller amplitudes and larger time delays. So, any combination of $\omega \tau$ will produce the same results: if one wants to measure signals with high frequency content, then a system with an appropriately small $\tau$ is required and vice versa (Figliola \& Beasley, 2011). 


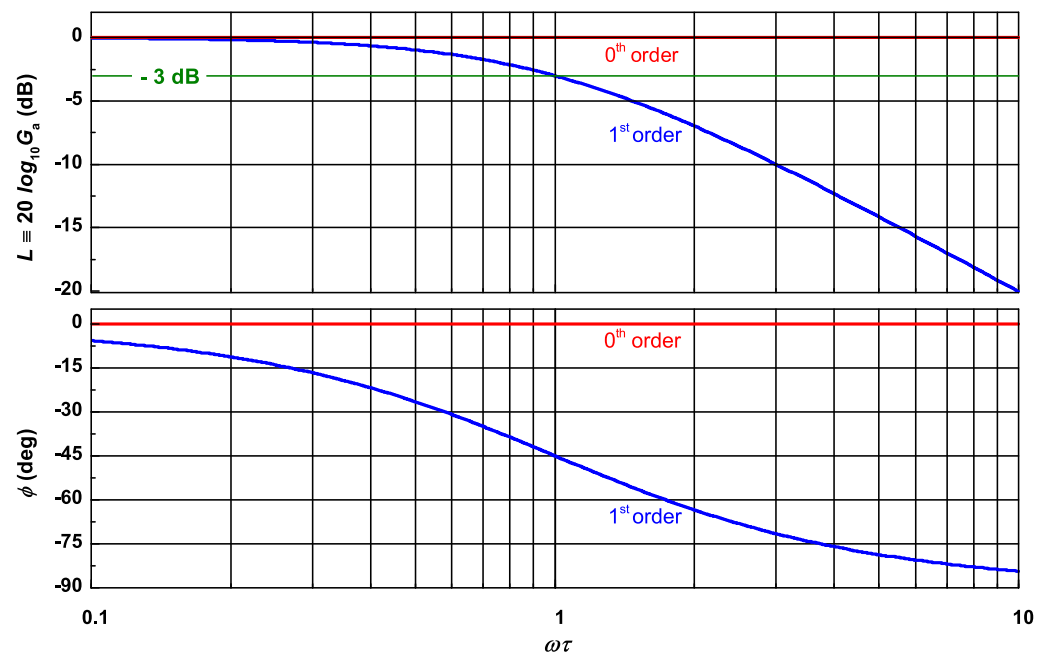

Fig. 13. Bode-diagram for zero- and first-order systems.

In a second-order system response, as shown in Fig. 14, at low values of $\omega / \omega_{n}$, values of $L(\omega)$ and $\phi(\omega)$ remain near to zero. This indicates that information concerning the input signal of frequency, $\omega$, will be passed through to the output with little attenuation and phase lag. This region of frequency response curves is called the transmission band. The actual extent of the frequency range for near unity gain depends on the system damping ratio, $\zeta$. The transmission band of a system is typically defined as $-3 \mathrm{~dB} \leqq L(\omega) \leqq 3 \mathrm{~dB}$. At large values of $\omega / \omega_{n}$, the system will attenuate the amplitude information of the input signal and a large phase shift occurs (Figliola \& Beasley, 2011). For a system with $\zeta \rightarrow 0, L(\omega)$ will be very high and $\phi(\omega) \rightarrow-180^{\circ}$ in the vicinity of $\omega / \omega_{n}=1$. This behaviour is a characteristics of

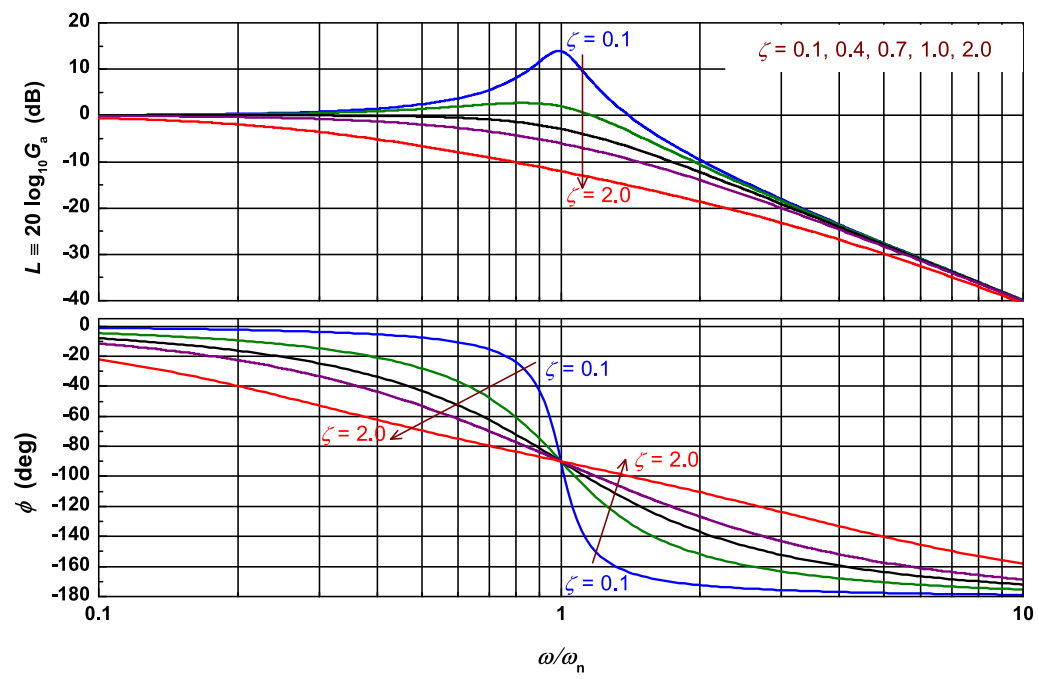

Fig. 14. Bode-diagram for second-order systems. 
system resonance. However, real systems possess some damping to limit the abruptness and magnitude of resonance, but underdamped systems will experience some resonance.

For second-order systems, response shown in Fig. 14, following observations can be made:

- System has a good linearity for low values of $\zeta$ and up to a frequency ratio, $\omega / \omega_{n}$, of about 0.3 as the amplitude gain is very nearly unity $\left(G_{a} \simeq 1\right)$ with $L(\omega) \simeq 0$.

- For large values of $\zeta$, the amplitude is reduced substantially.

- The phase shift characteristics are a strong function of $\omega / \omega_{n}$ for all frequencies.

- As a general rule of thumb, the choice of $\zeta=0.707$ is optimal since it results in the best combination of amplitude linearity and phase linearity over the widest range of frequencies (Holman, 2001).

The universal curves (Figs. 13 and 14) may be used as guidance in the selection of measurement instruments and system components. As the values of $L(\omega)$ and $\phi(\omega)$ deviate from zero, these curves take on rather steep slopes, and in these regions, small errors in the predictions of $\tau$ and the deviation of the real system from ideal one may lead to significant errors in measurements (Figliola \& Beasley, 2011).

\section{References}

ASME (2005). ASME PTC 19.1-2005: Test Uncertainty. Amarical Society of Mechanical Engineers (ASME), New York, USA.

Beckwith, T., Marangoni, R. \& Lienhard, J. (2007). Mechanical Measurements, 6th edn, Pearson Prentice Hall.

Doebelin, E. O. (1998). System Dynamics: Modeling, Analysis, Simulation, Design, Marcel Dekker, Inc. NY, USA.

Doebelin, E. O. (2004). Measurement Systems: Applications and Design, 5th edn, McGraw-Hill.

Dorf, R. \& Bishop, R. (1998). Modern Control Systems, 8th edn, Pearson Prentice Hall.

Dunn, P. F. (2010). Measurement and Data Analysis for Engineering and Science, 2nd edn, CRC Press.

Figliola, R. \& Beasley, D. (2011). Theory and Design for Mechanical Measurements, 5th edn, John Wiley.

Holman, J. (2001). Experimental Methods for Engineers, 7th edn, McGraw-Hill.

ISO (2007). ISO/IEC Guide 99:2007 International Vocabulary of Metrology - Basic and General Concepts and Associated Terms (VIM). International Organization for Standardization (ISO), Geneva, Switzerland.

ISO (2008). ISO/IEC Guide 98:2008 Uncertainty of Measurement - Guide to the Expression of Uncertainty in Measurement (GUM). International Organization for Standardization (ISO), Geneva, Switzerland.

Kelly, S. G. (2003). System Dynamics, Cengage Learning. 


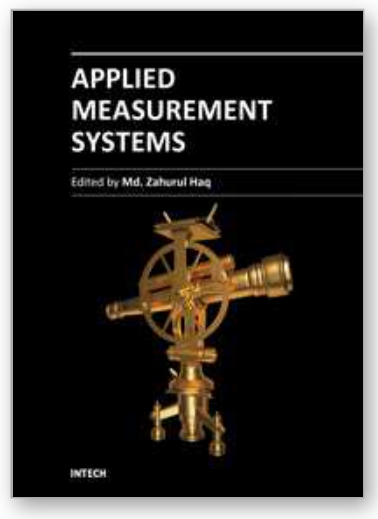

\author{
Applied Measurement Systems \\ Edited by Prof. Zahurul Haq
}

ISBN 978-953-51-0103-1

Hard cover, 390 pages

Publisher InTech

Published online 24, February, 2012

Published in print edition February, 2012

Measurement is a multidisciplinary experimental science. Measurement systems synergistically blend science, engineering and statistical methods to provide fundamental data for research, design and development, control of processes and operations, and facilitate safe and economic performance of systems. In recent years, measuring techniques have expanded rapidly and gained maturity, through extensive research activities and hardware advancements. With individual chapters authored by eminent professionals in their respective topics, Applied Measurement Systems attempts to provide a comprehensive presentation and in-depth guidance on some of the key applied and advanced topics in measurements for scientists, engineers and educators.

\title{
How to reference
}

In order to correctly reference this scholarly work, feel free to copy and paste the following:

Md. Zahurul Haq (2012). Measurement: System, Uncertainty and Response, Applied Measurement Systems, Prof. Zahurul Haq (Ed.), ISBN: 978-953-51-0103-1, InTech, Available from:

http://www.intechopen.com/books/applied-measurement-systems/measurement-system-uncertainty-andresponse

\section{INTECH}

open science | open minds

\section{InTech Europe}

University Campus STeP Ri

Slavka Krautzeka 83/A

51000 Rijeka, Croatia

Phone: +385 (51) 770447

Fax: +385 (51) 686166

www.intechopen.com

\section{InTech China}

Unit 405, Office Block, Hotel Equatorial Shanghai

No.65, Yan An Road (West), Shanghai, 200040, China 中国上海市延安西路65号上海国际贵都大饭店办公楼 405 单元

Phone: +86-21-62489820

Fax: $+86-21-62489821$ 
(C) 2012 The Author(s). Licensee IntechOpen. This is an open access article distributed under the terms of the Creative Commons Attribution 3.0 License, which permits unrestricted use, distribution, and reproduction in any medium, provided the original work is properly cited. 